\title{
The Effects of Errors in the Measurement of Continuous Exposure Variables on the Assessment of Risks
}

\author{
E. S. Gilbert
}

June 1988

Prepared for the U.S. Department of Energy under Contract DE-AC06-76RLO 1830

Pacific Northwest Laboratory Operated for the U.S. Department of Energy by Battelle Memorial Institute 


\title{
DISCLAIMER
}

This report was prepared as an account of work sponsored by an agency of the United States Government. Neither the United States Government nor any agency thereof, nor Battelle Memorial Institute, nor any or their employees, makes any warranty, expressed or implied, or assumes any legal liability or responsibility for the accuracy, completeness, or usefulness of any information, apparatus, product, or process disclosed, or represents that its use would not infringe privately owned rights. Reference herein to any specific commercial product, process, or service by trade name, trademark, manufacturer, or otherwise does not necessarily constitute or imply its endorseinent, recommendation, or favoring by the United States Government or any agency thereof, or Battelle Memorial Institute. The views and opinions of authors expressed herein do not necessarily state or reflect those of the United States Government or any agency thereof, or Battelle Memorial Institute.

\author{
PACIFIC NORTHWEST LABORATORY \\ operated by \\ BATTELLLE MEMORIAL INSTITUTE \\ for the \\ UNITED STATES DEPARTMENT OF ENERGY \\ under Contract DE-AC06-76RLO 1830
}

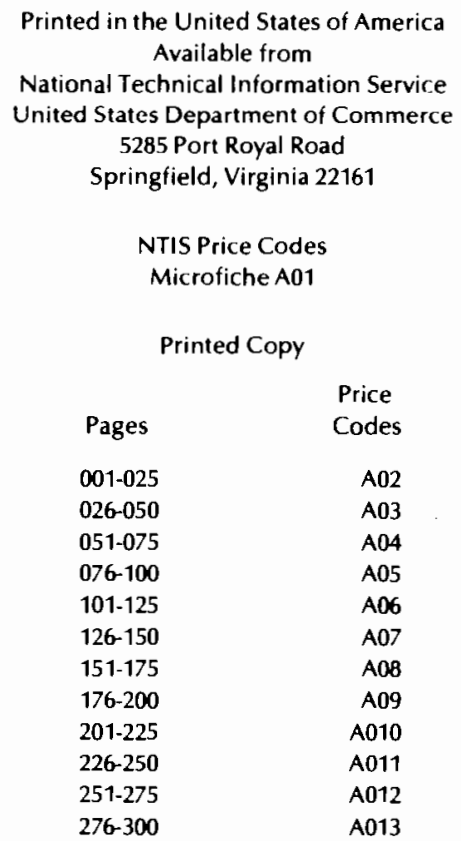




\section{2}

THE EFFECTS OF ERRORS IN THE MEASUREMENT OF CONTINUOUS EXPOSURE VARIABLES ON THE ASSESSMENT OF RISKS

E. S. Gilbert

June 1988

Prepared for the U.S. Department of Energy under Contract DE-AC06-76RLO 1830

Pacific Northwest Laboratory

Richland, Washington ' 99352 


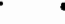


Exposure variables in epidemiological studies are seldom measured without error. However, it is unusual for such errors to be taken into account in analyzing data, and thus distortion of results may occur. These distorting effects are evaluated for the fitting of linear and log-linear proportional hazards models based on single continuous exposure variable, and are quantified under several sets of assumptions regarding the conditional distributions of the measured exposures given the true exposures, as well as assumptions regarding the true exposure distributions. For a wide range of assumptions, it is found that the most serious consequence of ignoring error is downward bias in the estimation of regression coefficients. In addition, the shape of the dose-response function may be distorted, and variances of estimated parameters may be underestimated. Except for the case of very large errors combined with highly skewed exposure distributions, tests of the null hypothesis of no effect that ignore error are found to be nearly as powerful as an optimal test, available if the error structure is known. 


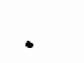




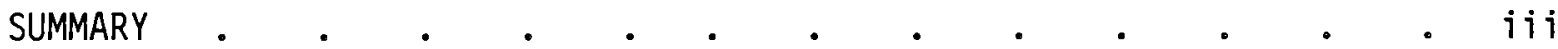

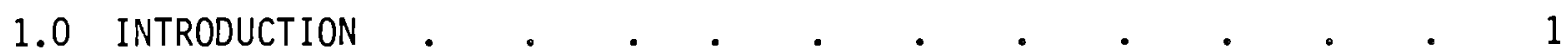

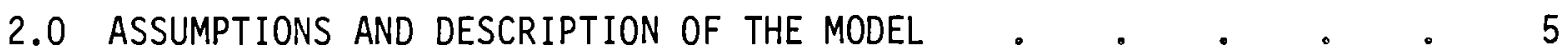

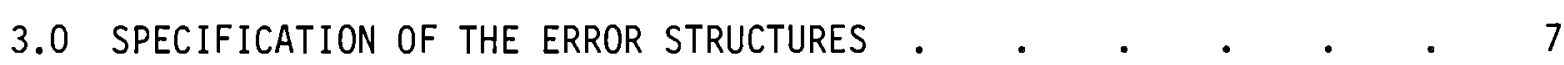

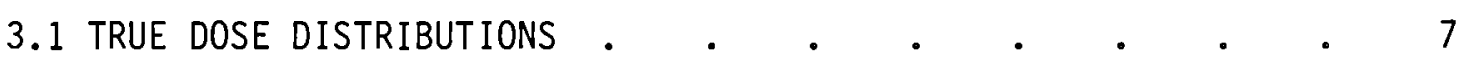

3.2 SPECIFICATION OF THE ERROR DISTRIBUTIONS $\quad$ • . $\quad$ • $\quad$. 10

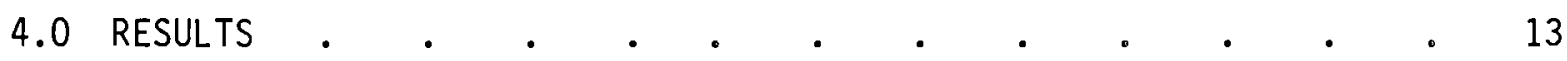

\begin{tabular}{l}
4.2 DISTORTION OF THE SHAPE OF THE DOSE RESPONSE \\
FUNCTION \\
\hline
\end{tabular}

4.3 BIAS IN THE ESTIMATED REGRESSION COEFFICIENTS . • • 17

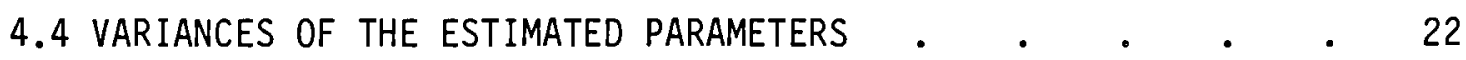

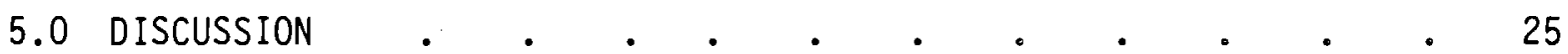

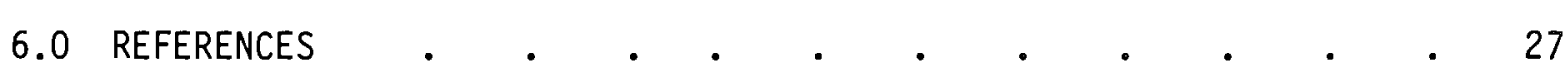

APPENDIX - TECHNICAL DETAIL AND COMPUTATIONAL PROCEDURES • • • $\quad$ A.1 


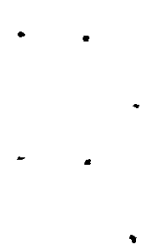




\section{FIGURES}

1 Probability Density Functions for the True Doses $z$. . . 8

$2 E(z ; x)$ Plotted as a Function of $x$ for Several Error Distributions. True Doses Follow a Lognormal Distribution with Coefficient of Variation of One . . . . . . . . 15

$3 \quad E(z ; x)$ Plotted as a Function of $x$ for Several Error Distributions. True Doses Follow a Lognormal Distribution with Coefficient of Variation of Two $. \quad . \quad . \quad . \quad . \quad . \quad 16$ 


\section{TABLES}

1 Properties of the Error Distributions . . . . . . . 11

2 Asymptotic Relative Efficiency of the Score Test Based on $x$ Relative to the Optimal Test Based on $E(z ; x)$ with Large and Moderate Amounts of Error . . . . 14

3 Factor $D$ by Which the Linear Regression Coefficient will be Biased Downward . . . . . . . . . . 19

4 Factor $D$ by Which the Linear Regression Coefficient will be Biased Downward When Unexposed Control is Included as Part of the True Dose Distribution . . . . 19

5 Factor $D$ by Which the Log-Linear Regression Coefficient will be Biased Downward . . . . . . . . . 20

6 Factor $D$ by Which the Log-Linear Regression Coefficient will be Biased Downward When Unexposed Control is Included as Part of the True Dose Distribution . . . $\quad$ • 20

7 Factor $D$ by Which the Linear Regression Coefficient will be Biased Downward with Doses Truncated at the g9th Percentile of the True Dose Distribution . . . $\quad 20$

8 Factor $D$ by Which the Log-Linear Regression Coefficient will be Biased Downward with Doses Truncated at the 99th Percentile of the True Dose Distribution . . . . $\quad 21$

9 Ratio of the Estimated and Correct Asymptotic Variances for Linear Mode1 . . . . . . . . . . . . 23

10 Ratio of Estimated and Correct Information for Log-

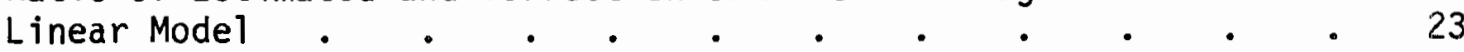

11 Ratio of the Estimated and Correct Asymptotic Variances for Linear Model with Unexposed Control Included as

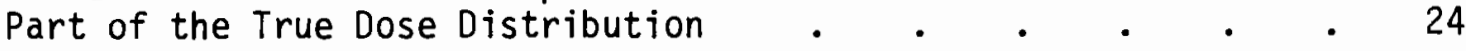

12 Ratio of the Estimated and Correct Asymptotic Variances for the Log-Linear Model with Unexposed Control Included as Part of the True Dose Distribution 


\section{THE EFFECTS OF ERRORS IN THE MEASUREMENT OF \\ CONTINUOUS EXPOSURE VARIABLES ON THE ASSESSMENT OF RISKS}

\subsection{INTRODUCTION}

It is well known that errors in the measurement of independent variables in ordinary linear regression can result in loss of power in testing for associations and may also lead to bias in the estimated regression coefficients (Cochran 1965, Kendall and Stuart 1979). It is also known that misclassification in dichotomous risk factors or exposure variables will lead to loss of power and bias in estimated relative risks of disease in epidemiological studies. This problem has been investigated by many, most recently by Flegal, Brownie, and Haas (1986), who provide an extensive list of references. The effects of errors in quantitative risk factors in estimating the risk of disease has not received as much attention, although Gladen and Rogan (1979) and Marshal et al. (1981) have examined the effect of misclassification in conducting trend tests based on several levels of a risk factor.

In this paper, we consider the situation in which interest centers on investigating the relationship of the risk of disease and a potentially harmful agent that has been measured on a continuous scale. An example is the study of Japanese atomic bomb survivors, where a major objective is the assessment of the dose-response relationship of cancer mortality and radiation dose. Gilbert (1984) examined the effects of errors in the measurement of these doses in making this assessment based on specific assumptions regarding the magnitude and distribution of the measurement errors as well as the distribution of the true doses. It was found that although the presence of measurement error would lead to loss of power in testing for an association of radiation dose and cancer mortality, the usual test statistics based on measured doses did nearly as well as tests that took account of the errors. It was also found that if the true dose-response function was linear, the observed dose-response function would not be linear, and that the estimated linear regression coefficients would be biased downward if measurement errors were not taken into account. 
Data from epidemiological studies of exposures to potentially harmful agents have traditionally been analyzed using methods that are strictly appropriate only if the exposures or doses are measured without error. The dose-response functions obtained from these studies are often used to develop understanding of disease mechanisms, and to predict risks in populations for whom future exposure is a possibility. It is the dose-response function based on the true doses that is needed for these purposes, and any distortion that may have come about because of dose measurement errors can potentially lead to faulty understanding and risk predictions. It is not known to what extent the results noted above for the atomic bomb survivors will generalize, but it is of interest to investigate the possible distortion of results when measurement error has not been taken into account.

The approach used in this paper is to consider several true dose distributions and several assumptions regarding the nature and magnitude of the errors in measuring these true doses. The distributions are hypothetical, but are chosen to approximate a range of possibilities likely to be encountered in actual epidemiological studies. The distortion in results arising if measurement error is not accounted for is then examined. Specifically, loss of power, distortion of the shape of the dose-response function, bias in the estimated regression coefficients, and the adequacy of asymptotic variance estimates are evaluated in the context of 1 inear (additive) and log-linear (multiplicative) proportional hazards models.

Recently, methods of analysis, based on the proportional hazards model, that take account of measurement error have been proposed by Prentice (1982). Whittemore and Grosser (1986) have also proposed a general method for accounting for errors as well as missing data. However, these methods require information on the error structure that may not be available, and tend to be computer intensive. Clayton (a) has also discussed various approaches for handling measurement error; the Clayton paper indicates clearly that this is a very difficult statistical problem, and that further research is needed to develop practical solutions.

(a) Clayton D. C. In Press. "Models for the Analys is of Cohort and Case-control Studies with Inaccurately Measured Exposure." In: Longitudinal Methods in Health Research. Oxford: Oxford University Press. 
The intention of this paper is not to provide solutions, but rather to develop awareness of an important problem. It is believed that a general understanding of the distortion that can come about because of measurement error will be helpful in interpreting results of analyses based on doses that have been measured with error, even if it is not possible to provide precise "corrections." It is, however, hoped that investigators will explore methods such as those noted above, and consider applying such methods to their data. 


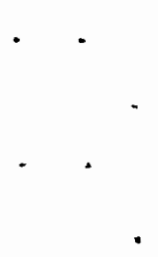




\subsection{ASSUMPTIONS AND DESCRIPTION OF THE MODEL}

It is assumed that all inferences are based either on the hazard function regression model originally proposed by Cox (1972) and given by

$$
\lambda(t ; z)=\lambda_{t} \exp \left(\beta z_{t}\right)
$$

or on a linear modification of this model discussed by Prentice and Mason (1986) and given by

$$
\lambda(t ; z)=\lambda_{t}\left(1+\beta z_{t}\right)
$$

In both cases, $t$ denotes time, $\lambda_{t}$ is an arbitrary baseline hazard, and $z_{t}$ denotes the dose at time $t$. These two models, which allow for the possibility of censoring, will be referred to as log-linear and linear dose-response models, respectively. It is assumed that tests of the null hypothesis are based on the score statistic arising from these models, and that estimates are obtained by solving the partial likelihood equations. These general procedures have been described elsewhere (Kalbfleisch and Prentice 1980), and are reviewed in the appendix.

Prentice (1982) has discussed the problem of making inferences about the hazard function based on the true exposures $z$ when only measured exposures $x$ are available, and has noted that meaningful inferences are possible only if it is assumed that measurement errors are independent of the occurrence of the event of interest. This assumption is made throughout this paper.

It is further assumed that the joint distribution of $x$ and $z$ does not change over the follow-up period. For fixed exposures (non-time-dependent), this assumption will probably be reasonably valid provided the hazard for the event of interest is small, and provided that the censoring mechanism is fairly independent of exposure. For time-dependent exposures, such as cumulative occupational exposure, the distribution of the true doses is likely to change with time. However, it is still believed that the approach taken here will provide useful insights regarding the impact of measurement errors on analyses. It is also assumed that the exposure is independent of other 
risk factors so that there is no need to control for possible confounding factors. Finally, it is assumed that all events occur at distinct failure times $t$ (no ties), and that the effect of exposure, if any, is to increase the risk of the disease of interest.

Prentice (1982) has shown that, in general, appropriate inferences based on the measured $x$ require knowledge of the conditional probability density functions $f(z ; x)$. The approach suggested by Prentice will be referred to as the "optimal procedure" in the material that follows. Additional discussion of this approach is given in the appendix. For the linear model, the optimal procedure requires replacing the measured doses $x$ by $E(z ; x)$, the expected value of the true dose giving rise to the measured $x$, while for the log-linear model, the optimal procedure requires replacing $\exp (\beta x)$ by $E[\exp (\beta z) ; x]$. For testing the null hypothesis that $\beta=0$, Prentice has shown that tests for both linear $\log -1$ inear models are obtained by replacing $x$ by $E(z ; x)$.

It may be of interest to note that if $z$ is a binomial variable, the replacement of $x$ by $E(z ; x)$ is the procedure suggested by Copeland et al. (1977) to correct for bias in estimating the relative risk due to misclassification. Applying Bayes Theorem, $E(z ; x)$ can be expressed as a function of the probability that $z=1$, and the sensitivity and specificity in measuring $z$. These parameters define the error structure, and must be known if the correction is to be applied. 


\subsection{SPECIFICATION OF THE ERROR STRUCTURES}

Although the discussion above indicates that it is the conditional distributions of the true doses $z$ given the measured doses $x$ that are required for appropriate inferences, understanding of the error structure in a given situation will frequently be in terms of the distributions of the measured doses $x$ conditional on the true doses $z$ as well as the marginal distributions of $x$ and/or $z$. In this paper, the error structures will be specified in terms of the probability densities functions for $x$ conditional on $z$, which will be referred to as the "error distributions" and the marginal probability densities of $z$, which will be referred to as the "true dose distributions."

\subsection{TRUE DOSE DISTRIBUTIONS}

It is assumed that the exposure of interest is a positive valued exposure (which will of ten be referred to as dose), with zero values representing no exposure from the source of interest. Several distributions from the lognormal and gamma families have been considered. The properties of these distributions are discussed by Johnson and Kotz (1970).

Both the lognormal and gamma families can be parametrized by a shape parameter and a scale parameter. The shape parameter alone determines the coefficient of variation. Results are presented for lognormal distributions with coefficients of variation of $0.1,1$, and 3 , and gamma distributions with coefficients of variation of 0.7 , and 2 . These density functions are plotted in Figure 1. Calculations were also made for the lognormal distribution with a coefficient of variation of 5 and a gamma distribution with coefficients of variations of 1 and 3 ; results of those additional calculations followed similar patterns to those presented. The lognormal and gamma distributions will be referred to as $L$ or $G$ respectively followed by the coefficient of variation. For example, the lognormal distribution with coefficient of variation of 1 will be referred to as LI. The lognormal distribution with coefficient of variation equal to 0.1 will be very close to a normal distribution, while the gamma distribution with coefficient of variation equal to one is an exponential distribution. 

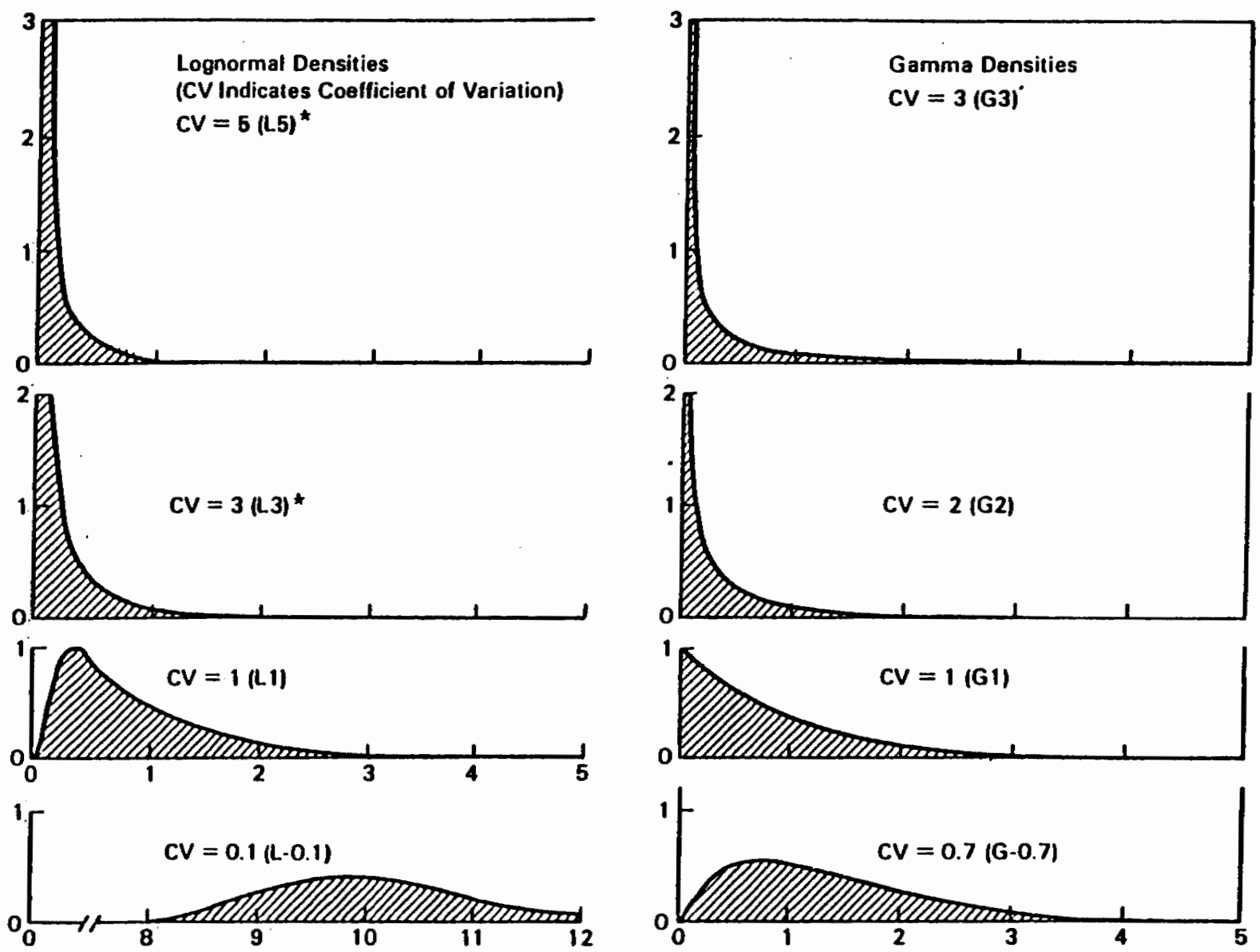

* These lognormal density functions approach 0 as $z$ approaches 0 , but this cannot be discerned when plotted on the scale here.

FIGURE 1. Probability Density Functions for the True Doses z 
Because there are generally practical limits to the values of the true exposures $z$, in most cases, the distributions specified above have been truncated at the upper 99th percentile. The truncated distributions have been scaled so that the variance is equal to one. In two cases, the lognormal distributions with a coefficient of variation of 1 (L1) and the gamma distribution with a coefficient of variation of 2 (G2), distributions truncated at the 99.99 th percentile were also considered, but results are presented only for $L 1$, and will be referred to as $L 1^{\prime}$.

These distributions were selected to allow examination of the impact of measurement error under several different assumptions regarding the true dose distributions. The distributions with smaller coefficients of variation may represent situations in which all subjects are "exposed" or have positive values. An occupational study in which all individuals are exposed to a substance but to different degrees would be an example. Variables such as blood pressure or serum cholesterol are other examples of variables following this pattern, although these are not ordinarily regarded as "exposures." The distributions with larger coefficients of variation may approximate situations where most subjects receive little or no exposure, while a few subjects receive large exposures. The Japanese atomic bomb survivors (Kato and Schul1 1982) and Hanford workers exposed to radiation (Gilbert 1985) follow this general pattern.

In addition to the distributions noted above, true dose distributions were considered in which half the doses were zero, with the other half conforming to one of the distributions considered above. This was done as a way of investigating the impact of error with an unexposed control group included. It was assumed that the exposures of the control were truly zero (measured without error). These true dose distributions will be designated by the symbol for the distribution comprising the exposed portion of the sample followed by "U" (for unexposed). For example, LIU denotes the true dose distribution in which half the doses follow a lognormal distribution (truncated at the 99 th percentile) with coefficient of variation equal to one, and half the doses are zero. 


\subsection{SPECIFICATION OF THE ERROR DISTRIBUTIONS}

For the error distributions, normal, gamma, and lognorma 1 distributions were considered. The normal distributions were parametrized so that $E(x ; z)=z$ and $\operatorname{Var}(x ; z)=h^{2}$, or $k^{2} z$, and will be referred to as error distributions $I$ and $I I$, respectively. The gamma distributions were parametrized so that $E(x ; z)=z$ and $\operatorname{Var}(x ; z)=k^{2} z$, and will be referred to as error distribution III. Finally, the lognormal densities were parametrized so $\log x$ given $z$ is normally distributed with mean $\log z$ and variance $s^{2}$. It then follows from properties of the lognormal distribution (Prentice and Mason 1986) that $E(x ; z)=w z$ and $\operatorname{Var}(x ; z)=w^{2}\left(w^{2}-1\right) z^{2}$, where $w=\exp \left(s^{2} / 2\right)$. This distribution will be referred to as error distribution IV. In all cases, it is assumed that the measurement errors for different subjects are independent of one another.

The properties of these error distributions are summarized in Table 1. Error distributions II, III, and IV reflect the common situation in which measurement errors increase with the size of the exposure. In particular, if the exposure is the sum of several measurements that are measured either with constant error or error in which the variance is proportional to the true exposure, then distributions such as II or III will result. Cumulative occupational exposures are usually obtained as the sum of estimated exposures for each of several time periods, and thus may be an example in which distributions II or III apply. Error distribution IV may provide a reasonable description of the measurement errors in doses for the Japanese atomic bomb survivors (Gilbert 1984, and Jablon 1971).

With the normal error distributions I and II, negative measurements are possible, and may be highly probable if true exposure values near zero have high probability. The usual practice in such cases would be to assign subjects with negative measured exposures values of zero. We shall also follow this practice, leading to the use of normal error distributions, truncated at zero.

An important property of the four error distributions is that $E(x ; z)=b z$ for $a 11 z$, where $b=1$ for error distributions I, II, and III, and $b=w$ for error distribution IV. Thus it is random error rather than systematic bias that is being addressed, although the lognormal error 


\section{TABLE 1. Properties of the Error Distributions}

\begin{tabular}{cccc}
$\begin{array}{l}\text { Error } \\
\text { Distribution }\end{array}$ & Type & $E(x ; z)$ & $\operatorname{Var}(x ; z)$ \\
\hline I & Normal & $z$ & $h^{2}$ \\
II & Norma 1 & $z$ & $k^{2} z$ \\
II & Gamma & $z$ & $k^{2} z$ \\
IV & Lognormal & $w z$ & $w^{2}\left(w^{2}-1\right) z^{2}$
\end{tabular}

distribution (IV) does induce some bias. The distortion resulting from systematic bias can be readily assessed provided the bias is known. The error distributions above do not include the situation in which an average value is assigned to each of a group of subjects and the measurement error consists of deviation of individual values from the assigned average. Under the assumption that $E(x ; z)=b z$, it is not difficult to show that $\operatorname{Var}(x / b)=$ $\operatorname{Var}(z)+E V$ where $E V=\int \operatorname{Var}(x / b ; z) g(z) d t$, and where $g(z)$ is the probability density function for the true doses $z$. EV will equal $h^{2}, k^{2} E(z), k^{2} E(z)$, and $\left(w^{2}-1\right) E\left(z^{2}\right)$ for error distributions I, II, III, and IV respectively.

Using these expressions, the values of $h, k$, and $s$ (or $w$ ) were determined so that $\operatorname{Var}(x / b)$ is either $100 \%$ or $20 \%$ larger than $\operatorname{Var}(z)$. The larger values were chosen to examine effects in a fairly extreme situation, while the smaller values are intended to represent more realistic amounts of error. These two cases will be referred to as "large" and "moderate" errors, respectively. In setting the values for $h$ and $k$, the truncation of observed negative values was not taken into account.

It is shown in the appendix that the choices for "large" and "moderate" amounts of error correspond to situations in which the asymptotic relative efficiency of the efficient score test (Kalbfleisch and Prentice 1980), based on the measured doses relative to that based on the true doses, is approximately $50 \%$, and $83 \%$, respectively. The asymptotic relative efficiency can be thought of as the limiting relative efficiency as the sample size 
increases and the regression coefficient $B$ approaches zero; the relative efficiency is the reciprocal of the ratio of the sample sizes required to attain a given power. 


\subsection{RESULTS}

Calculations have been made to allow quantification of the distorting effects of measurement error in the fitting of linear and $10 \mathrm{~g}-1$ inear proportional hazards models. The error structures considered for these calculations include most of the possible combinations of the true dose distributions and error distributions defined above. The methods used for these calculations are described in detail in the appendix.

\subsection{ASYMPTOTIC RELATIVE EFFICIENCY OF TESTS OF THE NULL HYPOTHESIS}

It was noted above that the asymptotic relative efficiency for the score test based on $x$ relative to that based on $z$ will be less than one, and that for the error structures considered in this paper was set to be approximately 0.500 and 0.833 . Since the option of basing tests on true doses is not usually available, a question of more practical interest than the comparison of the power of tests based on $x$ and $z$, is the comparison of the power of tests based on $x$ with tests based on $E(z ; x)$, the optimal procedure if the $z$ are unavailable, but the error structure can be specified. That is, what is to be gained by the additional effort of taking error into account?

The asymptotic relative efficiency of the score test based on $x$ relative to the optimal test has been evaluated for each of the error structures described above with the results presented in Table 2. With moderate errors, the asymptotic relative efficiency never drops below $90 \%$, and only occasionally does it drop below 95\%. With large errors, the asymptotic relative efficiency usually exceeds $90 \%$, but sometimes falls below this value. In general, the asymptotic relative efficiency is lowest for the lognormal error distributions, and highest for the error distributions II and III, where the variance of the estimated doses conditional on the true doses has been assumed to be proportional to the true doses. The asymptotic relative efficiency is also smaller for the more highly skewed true dose distributions (with large coefficients of variation). The asymptotic relative efficiency based on analyses in which a control (with doses of zero and no measurement error) is included has also been evaluated for each of the cases presented in Table 2, but is not shown here. These calculations also indicate good performance of tests based on $x$ relative to those based on $E(z ; x)$. 
TABLE 2. Asymptotic Relative Efficiency of the Score Test Based on $x$ Relative to the Optimal Test Based on $E(z ; x)$ with Large and Moderate Amounts of Error*.

Error distribution True dose distribution

$\begin{array}{lllllll} & L-.1 & L 1 & L 3 & G-.7 & G 2 & \text { L1' } \\ \text { Moderate errors } & & & & & & \\ \text { I } & 1.00 & 0.98 & 0.97 & 0.99 & 0.97 & 0.97 \\ \text { I I } & 1.00 & 0.99 & 0.99 & 1.00 & 0.99 & 0.99 \\ \text { I I } & 1.00 & 1.00 & 0.99 & 1.00 & 0.99 & 1.00 \\ \text { IV } & 1.00 & 0.98 & 0.96 & 0.99 & 0.95 & 0.99 \\ \text { Large errors } & & & & & & \\ \text { I } & 1.00 & 0.92 & 0.86 & 0.97 & 0.90 & 0.86 \\ \text { I I } & 0.99 & 0.97 & 0.97 & 0.99 & 0.98 & 0.95 \\ \text { I I } & 1.00 & 0.97 & 0.93 & 0.95 & 0.83 & 0.97 \\ \text { IV } & 0.99 & 0.86 & 0.77 & 0.84 & 0.72 & 0.90\end{array}$

* Table entries can also be interpreted as the squared correlation coefficient of $x$ and $E(z ; x)$.

\subsection{DISTORTION OF THE SHAPE OF THE DOSE RESPONSE FUNCTION}

Figures 2 and 3 investigate the distortion of the shape of a linear dose response function that is likely to come about because of measurement error. In these figures, $E(z ; x)$ is plotted as a function of $x$. Since, the excess risk based on the true doses is given by $\lambda_{t} \beta z$, while that based on the measured doses is given by $\lambda_{t} \beta E(z ; z)$, these plots can be interpreted as the ratio of the response that would be expected when the doses are measured with moderate and large amounts of error to the response when the doses are measured without error.

It is seen that even though the response based on the true doses is linear, none of the responses based on doses measured with error are exactly linear. The departures from the true dose response function comes about because measured doses close to zero often truly represent larger doses, and thus the observed response is higher than it should be, while large measured doses often truly represent smaller doses so that the observed response is lower than in should be.

As is noted in the appendix, the asymptotic relative efficiency, presented in Table 2, can also be interpreted as a measure of the departure 

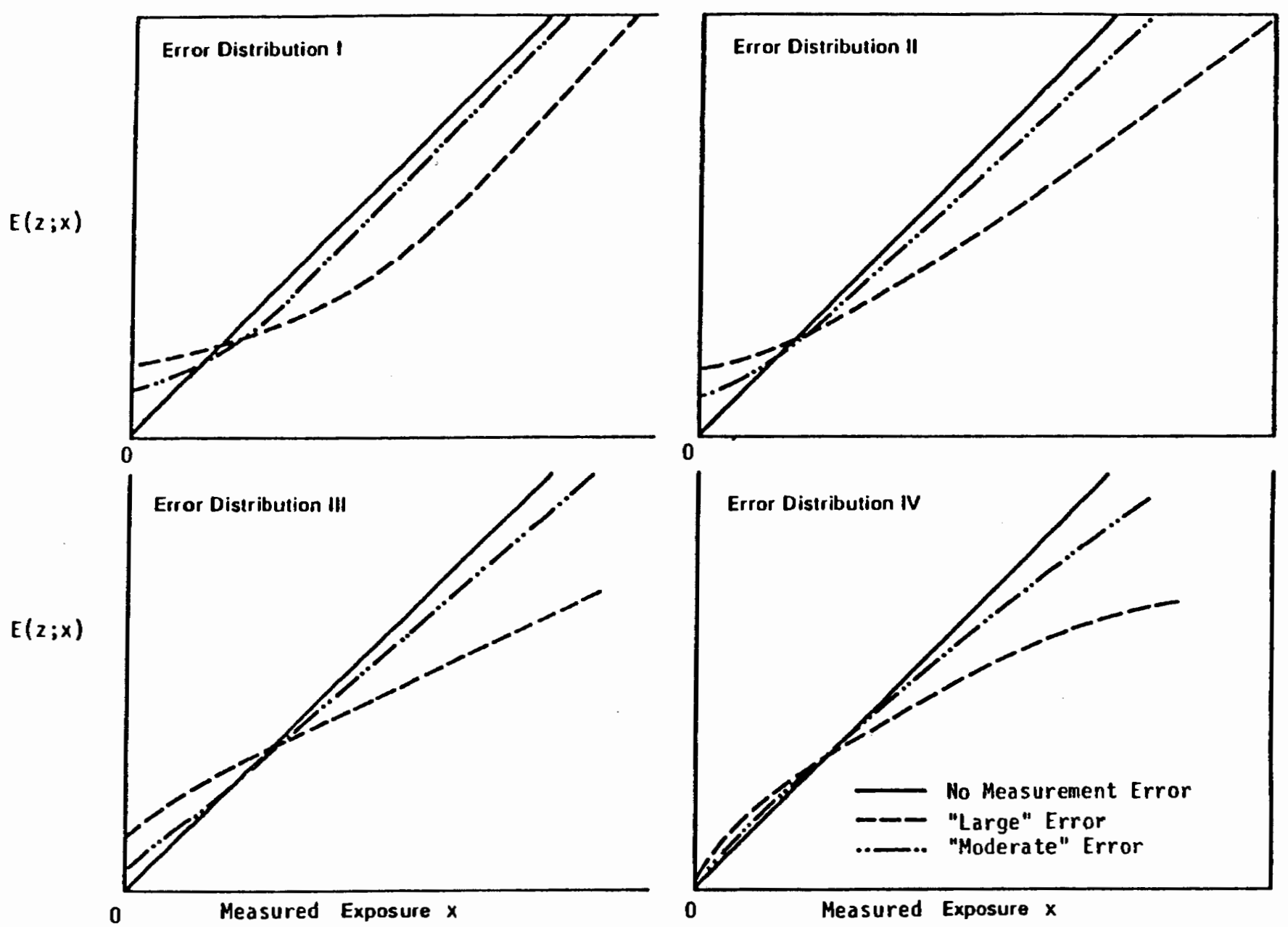

FIGURE 2. $E(z ; x)$ Plotted as a Function of $x$ for Several Error Distributions. True Doses Follow a Lognormal Distribution with Coefficient of Variation of One (L1)*

* These plots can be regarded as the expected observed response relative to the true linear response 

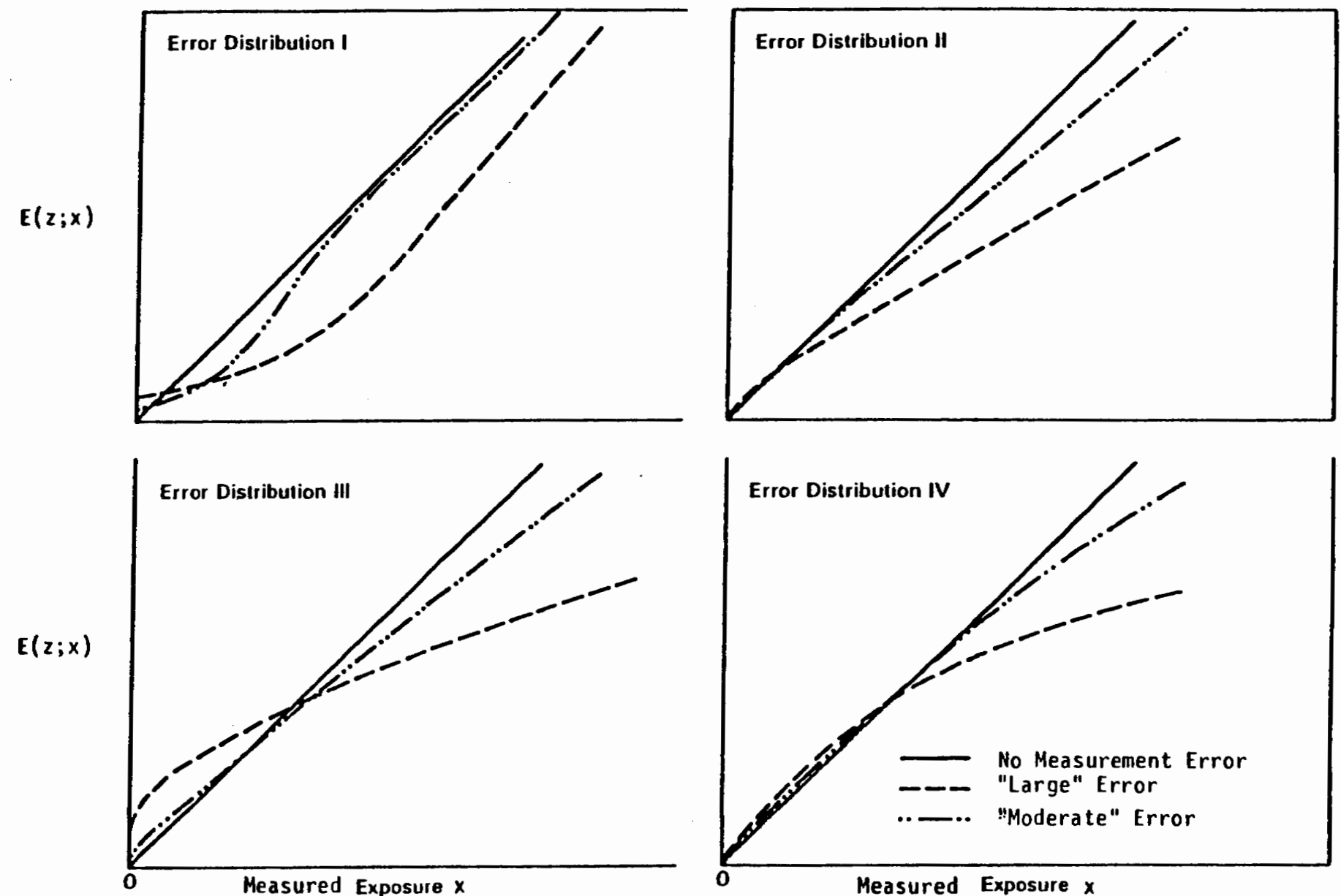

FIGURE 3. E $(z ; x)$ Plotted as a Function of $x$ for Several Error Distributions. True Doses Follow a Lognormal Distribution with Coefficient of Variation of Two (G2)*

* Those plots can be regarded as the expected observed response relative to the true linear response. 
from 1 inearity. It is seen that the greatest departures from linearity are for error distribution IV, and the least from distributions II and III. Similar plots to those presented in Figures 2 and 3 have also been made for the other true dose distributions considered. These plots are similar in general shape to those in Figures 2 and 3 although some structures show greater departures than others as reflected in the results presented in Table 2. The shape of the dose-response function appears to depend more on the error distribution than on the true dose distribution, and the skewed error distributions III and IV always induced convexity in the observed function.

For the $\log -1$ inear dose response function, plots of $\log [E\{\exp (\beta z ; x)\}] / B$ versus $x$ have been examined. These plots indicate that on a log scale, the distortion of the $\log -1$ inear dose response is similar to the distortion in the linear dose-response.

\subsection{BIAS IN THE ESTIMATED REGRESSION COEFFICIENTS}

If linear functions were fit to the responses based on large or moderate error shown in Figures 2 and 3 , it is evident that the slope would be less than that of the true linear responses. In this section, the magnitude of bias is determined that will result when linear and log-linear proportional hazards models are fit using maximum likelihood estimation, but with measured doses substituted for the true ones. In Tables 3-8, values of $D$ are presented such that $E(\hat{\beta})=D \beta$ where $\hat{B}$ and $\beta$ are the respective estimated and true regression coefficients. The values of $D$ will be referred to as "deflation factors." The true regression coefficients, $\beta$, have been set so that the relative risk at the 90 th percentile of the true dose distribution is either two or five. For results in which an unexposed control is included as a part of the true dose distribution, the same coefficient values have been considered as when no such control in included.

Cochran (1965) has shown that for ordinary linear regression, the resulting regression coefficient will be biased downward by a factor that is equal to the ratio of $\operatorname{Cov}(z, x)$ and $\operatorname{Var}(x)$, which, if it is assumed that $E(x ; z)=b z$ (where $b$ is a constant) for all $z$, can be shown to equal

$B=b \operatorname{Var}(z) / \operatorname{Var}(x)$. Cochran's result has been applied to the linear 
proportional hazards model (Prentice 1982) to suggest a biasing factor of $C=B /(1+B A)$, where $A=E(z)(1-b B)$. The factor in the denominator for the expression for $C$ results because the baseline risk at low or zero doses tends to be overestimated by the factor $1+B A$. It should be noted that the above expressions will not hold exactly for fitting the linear proportional hazards based on maximum likelihood estimation, and this is the reason the calculations discussed below are needed.

The error parameters $h, k$, and $w$ have been set so that $b B$ would be approximately $50 \%$ for "large" errors, and approximately $83.3 \%$ for moderate errors. However, the factor $1 /(1+B A)$ will depend on the value of $B$ and on the mean of the true dose distribution. This explains to a large extent the fact that the deflation factors presented in Tables 3 and 4 for the linear model are lowest for the true dose distributions with larger means (see Figure 1). It also explains the increase in the deflation factors when a control included. This increase is particularly notable with the L-0.1 distribution. This distribution is close to a normal distribution with mean 10 and variance 1. The absence of bias when a control is introduced apparently results because the estimated coefficient depends almost entirely on the difference in "exposed" individuals and controls, with the variability among the exposed playing an insignificant role. Thus as long as the average dose can be estimated correctly, there is little bias.

With the log-linear model (Tables 5 and 6 ), results do not depend as strongly on the true dose distributions or the inclusion of a control as with the linear model. Note that if $A$ and $B$ have been determined so that $\exp (A+B x)$ approximates $E[\exp (B z) ; x]$, then the factor $\exp (A)$ will be absorbed in the spontaneous risk portion of the hazard function so that a positive value for $A$ does not lead to further deflation of the relative risk coefficient as happens with the linear model. With the log-linear model, the lowest deflation factors are generally observed with the more highly skewed true dose distributions and with error distribution IV. In these cases, extremely large measured doses, which severely overestimate the true doses, will occur with positive probability. With a relative risk function that is exponential, the relative risks for these overestimated doses may be extremely large, leading to severe distortion of results. 
TABLE 3. Factor D by which the Linear Regression Coefficient will be Biased Downward

Relative risk at 90 th percentile Relative risk at 90 th percentile set at 2.0 set at 5.0

Error Distribution True dose distribution True dose distribution

Moderate errors

L-.1 L1 $\quad$ L3 $\quad$ G-.7 $\quad$ G2 $\quad$ L1' $1^{\prime} \quad$ L-.1 $\quad$ L1 $\quad$ L3 $\quad$ G-.7 $\quad$ G2 $\quad$ L1'

$\begin{array}{lllllllllllll}\text { I } & 0.72 & 0.76 & 0.82 & 0.76 & 0.89 & 0.73 & 0.52 & 0.57 & 0.66 & 0.59 & 0.82 & 0.53 \\ \text { II } & 0.72 & 0.76 & 0.78 & 0.77 & 0.82 & 0.73 & 0.52 & 0.60 & 0.66 & 0.62 & 0.77 & 0.55 \\ \text { II I } & 0.72 & 0.76 & 0.77 & 0.77 & 0.82 & 0.74 & 0.52 & 0.62 & 0.65 & 0.64 & 0.76 & 0.57 \\ \text { IV } & 0.72 & 0.78 & 0.81 & 0.78 & 0.82 & 0.77 & 0.52 & 0.70 & 0.78 & 0.70 & 0.83 & 0.68 \\ \text { Large errors } & & & & & & & & & & & & \\ \text { II } & 0.35 & 0.46 & 0.53 & 0.44 & 0.59 & 0.44 & 0.18 & 0.29 & 0.37 & 0.28 & 0.47 & 0.27 \\ \text { II } & 0.34 & 0.43 & 0.49 & 0.40 & 0.53 & 0.42 & 0.18 & 0.27 & 0.35 & 0.25 & 0.42 & 0.25 \\ \text { III } & 0.34 & 0.42 & 0.46 & 0.42 & 0.51 & 0.40 & 0.18 & 0.28 & 0.35 & 0.28 & 0.44 & 0.25 \\ \text { IV } & 0.35 & 0.40 & 0.46 & 0.40 & 0.47 & 0.39 & 0.18 & 0.30 & 0.42 & 0.29 & 0.48 & 0.29\end{array}$

TABLE 4. Factor $D$ by Which the Linear Regression Coefficient will be Biased Downward When Unexposed Control is Included as Part of the True Dose Distribution

Relative risk at 90th percentile Relative risk at 90th percentile set at 2.0 set at 5.0

Error Distribution True dose distribution True dose distribution Moderate errors L-..1U LIU L3U G-.7U G2U LIU' L-.IU LIU L3U G-.7U G2U LIU'

$\begin{array}{lllllllllllll}\text { I } & 1.00 & 0.88 & 0.84 & 0.91 & 0.88 & 0.85 & 1.00 & 0.83 & 0.76 & 0.87 & 0.83 & 0.79 \\ \text { II } & 1.00 & 0.89 & 0.84 & 0.91 & 0.86 & 0.86 & 1.00 & 0.86 & 0.79 & 0.90 & 0.84 & 0.82 \\ \text { III } & 1.00 & 0.89 & 0.83 & 0.92 & 0.86 & 0.86 & 1.00 & 0.87 & 0.79 & 0.91 & 0.85 & 0.84 \\ \text { IV } & 1.00 & 0.88 & 0.85 & 0.90 & 0.85 & 0.87 & 1.00 & 0.89 & 0.86 & 0.91 & 0.87 & 0.88\end{array}$


TABLE 5. Factor $D$ by which the Log-Linear Regression Coefficient will be Biased Downward

Relative risk at 90th percentile Relative risk at 90th percentile set at 2.0 set at 5.0

Error Distribution True dose distribution True dose distribution Moderate errors

$\begin{array}{lllllllllllll}\text { I } & 0.83 & 0.90 & 0.96 & 0.87 & 0.98 & 0.98 & 0.83 & 0.93 & 0.92 & 0.89 & 0.95 & 0.91 \\ \text { II } & 0.83 & 0.84 & 0.83 & 0.83 & 0.82 & 0.91 & 0.83 & 0.84 & 0.69 & 0.82 & 0.74 & 0.82 \\ \text { III } & 0.83 & 0.84 & 0.81 & 0.83 & 0.80 & 0.91 & 0.83 & 0.83 & 0.67 & 0.83 & 0.70 & 0.81 \\ \text { IV } & 0.83 & 0.76 & 0.60 & 0.77 & 0.64 & 0.69 & 0.83 & 0.68 & 0.38 & 0.80 & 0.44 & 0.49 \\ \text { Large errors } & & & & & & & & & & & & \\ \text { I } & 0.50 & 0.66 & 0.86 & 0.59 & 0.81 & 0.89 & 0.50 & 0.75 & 0.83 & 0.63 & 0.84 & 0.88 \\ \text { II } & 0.50 & 0.57 & 0.59 & 0.51 & 0.56 & 0.73 & 0.50 & 0.58 & 0.46 & 0.51 & 0.48 & 0.63 \\ \text { III } & 0.50 & 0.51 & 0.49 & 0.49 & 0.46 & 0.67 & 0.50 & 0.50 & 0.36 & 0.48 & 0.36 & 0.60 \\ \text { IV } & 0.49 & 0.35 & 0.16 & 0.39 & 0.19 & 0.29 & 0.49 & 0.25 & 0.09 & 0.32 & 0.11 & 0.19\end{array}$

TABLE 6. Factor $D$ by which the Log-Linear Regression Coefficient will be Biased Downward When Unexposed Control is Included as Part of the True Dose Distribution

Relative risk at 90th percentile Relative risk at 90th percentile set at 2.0 set at 5.0

Error Distribution True dose distribution True dose distribution Moderate errors

$\begin{array}{lllllllllllll}\text { I } & 0.99 & 0.92 & 0.95 & 0.92 & 0.97 & 0.98 & 0.99 & 0.94 & 0.93 & 0.92 & 0.95 & 0.92 \\ \text { II } & 0.99 & 0.89 & 0.84 & 0.90 & 0.84 & 0.92 & 0.99 & 0.87 & 0.72 & 0.88 & 0.77 & 0.84 \\ \text { III } & 0.99 & 0.88 & 0.83 & 0.90 & 0.82 & 0.92 & 0.99 & 0.86 & 0.70 & 0.88 & 0.73 & 0.82 \\ \text { IV } & 0.99 & 0.81 & 0.61 & 0.85 & 0.66 & 0.71 & 0.99 & 0.81 & 0.41 & 0.80 & 0.46 & 0.51\end{array}$


TABLE 7. Factor $D$ by Which the Linear Regression Coefficient will be Biased Downward with Doses Truncated at the 99 th Percentile of the True Dose Distribution

Relative risk at 90th percentile Relative risk at 90th percentile set at 2.0 set at 5.0

Error

Distribution

True dose distribution

True dose distribution

Moderate errors

L-.1 L1 L L3 $\quad$ G-.7 G2 $\quad$ L1' $\quad$ L-.1 L1 $\quad$ L3 $\quad$ G-.7 $\quad$ G2 $\quad$ L1'

I 0.73

I I

I I I

0.73

$\begin{array}{lll}0.76 & 0.82\end{array}$

$0.77 \quad 0.90$

0.77

0.53

0.57

0.67

$\begin{array}{lll}0.59 & 0.82 & 0.55\end{array}$

0.76

0.79

0.78

$\begin{array}{ll}0.84 & 0.78\end{array}$

0.53

0.77

0.78

0.79

0.84

0.79

0.53

$0.60 \quad 0.66$

0.63

0.77

0.620 .66

0.65

$0.78 \quad 0.61$

IV

0.73

$\begin{array}{lllll}0.81 & 0.84 & 0.81 & 0.88 & 0.87\end{array}$

0.53

$0.73 \quad 0.82$

0.73

$0.87 \quad 0.79$

TABLE 8. Factor $D$ by which the Log-Linear Regression Coefficient will be Biased Downward with Doses Truncated at the 99th Percentile of the True Dose Distribution

Relative risk at 90 th percentile Relative risk at 90th percentile set at 2.0 set at 5.0

Error Distribution True dose distribution True dose distribution Moderate errors L-.1 L1 L3 L-.7 G2 $\quad$ L1' $\quad$ L-.1 $\quad$ L1 $\quad$ L3 $\quad$ G-.7 $\quad$ G2 $\quad$ L1' I 0.83

I I

I I I

0.83

0.90

$\begin{array}{lll}0.99 & 0.88 & 1.01\end{array}$

1.25

$\begin{array}{lll}0.86 & 0.90 & 0.85\end{array}$

0.83

0.86

$0.89 \quad 0.85$

0.901 .20

0.84

$0.94 \quad 1.01$

0.84

$\begin{array}{lll}0.89 & 1.19 & 0.84\end{array}$

0.83

$0.83 \quad 0.84$

0.84

$\begin{array}{lll}0.87 & 1.18 & 0.84\end{array}$

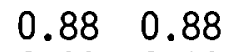

0.91

$\begin{array}{ll}1.06 \quad 2.90 \\ 0.93 & 2.82\end{array}$

$\begin{array}{ll}0.88 & 0.88 \\ 0.83 & 0.80\end{array}$

0.86

0.86

$0.93 \quad 2.82$

IV

$$
0.83
$$

\begin{abstract}
(
\end{abstract}
0.83

$\begin{array}{ll}0.80 & 0.83\end{array}$

$0.92 \quad 2.81$

$0.88 \quad 2.69$ 
Investigators often suspect that very large measured doses have been overestimated and either exclude these data points or reduce the estimated doses. For example, in the atomic bomb survivor studies, it is thought to be very unlikely that a person could initially survive a dose of 600 rad or more. Thus in analyses based on data from this study, measured doses exceeding this value have generally been set at $600 \mathrm{rad}$, and this procedure probably does serve to reduce bias in the estimated regression coefficients (Gilbert 1984).

To examine the effect of this practice, calculations were conducted in which all measured doses exceeding the 99 th percentile of the original lognormal and gamma true dose distributions were set to this value. Since most of the true dose distributions were truncated at the 99 th percentile, this will represent the maximum possible dose in these cases. Results of these calculation are given in Tables 7 and 8 . In general, this procedure results in a reduction in bias, particularly for the log-linear model. It must however be recognized that the choice of the truncation point was based on knowledge of the maximum true dose, and that one would not generally have certain knowledge of such a dose. Truncating at too low a value could serve to bias results in the opposite direction, as is demonstrated in the results for the LI' distribution.

\subsection{VARIANCES OF THE ESTIMATED PARAMETERS}

The adequacy of the inverse expected information in forming confidence limits for the deflated regression coefficient $D B$ has also been investigated. In Tables 9-12, the ratios of "estimated" and "correct" asymptotic variances are presented. The "estimated" asymptotic variance is that obtained from the likelihood based on $x$, taking no account of error, while the "correct" asymptotic variance is that obtained from the likelihood of Prentice multiplied by $D^{2}$.

For the linear model with no unexposed control, the asymptotic variances of the estimated parameters are generally underestimated. For the log-linear model, the asymptotic variance appears to be more appropriate, but with the more highly skewed true dose distributions, the variance is underestimated in this case as we11. The inclusion of a control greatly improves the adequacy of the asymptotic variance for the linear model. 
Even without measurement error, the use of the inverse information for determining confidence limits may not perform well (Prentice and Mason 1986), especially for the linear model. It has been recommended (Thomas 1981) that the likelihood ratio statistic be used to form confidence intervals in these cases. An investigation of the behavior of the likelihood ratio statistic with errors in the covariates would be of interest.

TABLE 9. Ratio of the Estimated and Correct Asymptotic Variances for Linear Model

Relative risk at 90th percentile Relative risk at 90th percentile set at 2.0 set at 5.0

Error

Distribution True dose distribution True dose distribution Moderate errors $\begin{array}{llllllllllll}\text { L-.1 } & \text { L1 } & \text { L3 } & \text { G-.7 } & \text { G2 } & \text { L1' } & \text { L-.1 } & \text { L1 } & \text { L3 } & \text { G-.7 } & \text { G2 } & \text { L1' }\end{array}$ I II I I I IV

0.76

0.82

$\begin{array}{lll}0.76 & 0.84 & 0\end{array}$

$0.89 \quad 0.83$

$0.97 \quad 0.78$

0.39

$\begin{array}{ll}0.47 & 0.61 \\ 0.52 & 0.67\end{array}$

$0.49 \quad 0.83$

0.39

0.76

0.85

0.89

$\begin{array}{lll}0.86 & 0.95 & 0.80\end{array}$

$\begin{array}{lll}0.76 & 0.94 & 1.01\end{array}$

$\begin{array}{lll}0.94 & 1.04 & 0.93\end{array}$

0.39

0.52

0.67

$\begin{array}{ll}0.56 & 0.80\end{array}$

$0.56 \quad 0.65$

$\begin{array}{ll}0.60 & 0.84\end{array}$

0.39

0.740 .91

0.750 .91

0.41

0.45

0.49

0.72

TABLE 10. Ratio of Estimated and Correct Information for Log-Linear

Relative risk at 90th percentile Relative risk at 90th percentile set at 2.0 set at 5.0

Error Distribution True dose distribution True dose distribution Moderate errors

$\begin{array}{lllllllllllll}\text { I } & 1.00 & 1.06 & 1.01 & 1.03 & 1.05 & 1.09 & 1.00 & 1.04 & 0.86 & 1.04 & 0.92 & 0.60 \\ \text { I I } & 1.00 & 1.03 & 1.01 & 1.01 & 1.03 & 1.08 & 1.00 & 1.01 & 0.57 & 1.00 & 0.94 & 0.32 \\ \text { I I } & 1.00 & 1.03 & 1.04 & 1.01 & 1.04 & 1.09 & 1.00 & 1.01 & 0.58 & 1.01 & 0.81 & 0.48 \\ \text { IV } & 1.00 & 1.02 & 0.87 & 1.01 & 0.96 & 0.97 & 1.00 & 0.93 & 0.44 & 0.97 & 0.61 & 0.30\end{array}$


TABLE 11. Ratio of the Estimated and Correct Asymptotic Variances for Linear Model with Unexposed Control Included as Part of the True Dose Distribution

\begin{tabular}{|c|c|c|c|c|c|c|c|c|c|c|c|c|}
\hline Mode & $\begin{array}{l}L-.1 U \\
\text { errors }\end{array}$ & LIU & L3U & $G-.7 U$ & G2U & L1U' & $L-.1 U$ & LIU & L $3 U$ & $G-.7 U$ & G2U & LIU' \\
\hline $\begin{array}{l}\text { I } \\
\text { I I } \\
\text { I I I } \\
\text { IV }\end{array}$ & $\begin{array}{l}1.00 \\
1.00 \\
1.00 \\
1.00\end{array}$ & $\begin{array}{l}0.99 \\
1.00 \\
1.01 \\
1.03\end{array}$ & $\begin{array}{l}0.94 \\
0.97 \\
0.98 \\
1.04\end{array}$ & $\begin{array}{l}0.99 \\
1.01 \\
1.01 \\
1.03\end{array}$ & $\begin{array}{l}1.97 \\
1.00 \\
1.02 \\
1.06\end{array}$ & $\begin{array}{l}0.97 \\
0.99 \\
1.00 \\
0.97\end{array}$ & $\begin{array}{l}1.00 \\
1.00 \\
1.00 \\
1.00\end{array}$ & $\begin{array}{l}0.93 \\
0.96 \\
0.98 \\
1.01\end{array}$ & $\begin{array}{l}0.87 \\
0.90 \\
0.92 \\
1.01\end{array}$ & $\begin{array}{l}0.96 \\
0.98 \\
0.99 \\
1.01\end{array}$ & $\begin{array}{l}0.92 \\
0.95 \\
0.98 \\
1.03\end{array}$ & $\begin{array}{l}0.91 \\
0.94 \\
0.96 \\
1.01\end{array}$ \\
\hline
\end{tabular}

TABLE 12. Ratio of Estimated and Correct Asymptotic Variances for the Log-Linear Model with Unexposed Control Included as Part of the True Dose Distribution

Relative risk at 90 th percentile Relative risk at 90th percentile set at 2.0 set at 5.0

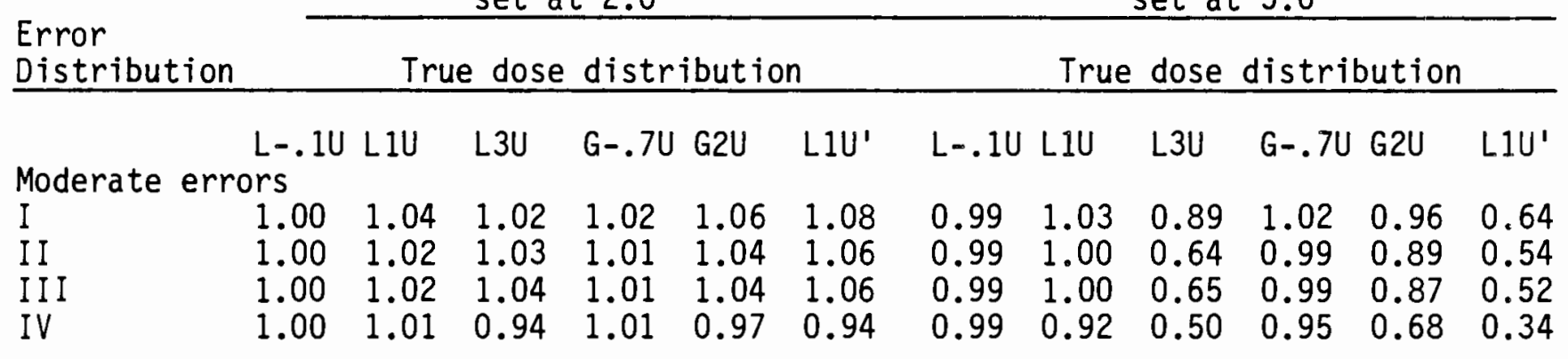




\subsection{DISCUSSION}

For a wide variety of error structures, it has been shown that if measurement error is not taken into account, distortion of the shape of the dose-response function, underestimation of linear and $10 \mathrm{l}-1$ inear regression coefficients, and underestimation of the asymptotic variances may result. The magnitude of these effects is not easily predicted, but will depend on the nature of the true dose distribution, the magnitude of the true relative risk coefficient, and the nature and magnitude of the measurement errors.

In general, simple formulae for correcting these distortions are not available. Correct inferences require first specifying the error structure, and second, determining the functions $E(z ; x)$ or $E[\exp (\beta z) ; x]$ needed for the application of methods such as those suggested by Prentice. Except in a few special cases, neither of these tasks is easily accomplished. By learning as much as possible about the dose estimation process, it may be possible to formulate reasonable assumptions regarding the error distributions, but it is not in general straight-forward to obtain the required densities $f(z ; x)$ based on the observed $x$ and knowledge of $g(x ; z)$. Clayton (a) has suggested methods for addressing this problem, but further research is needed before these methods can be generally applied.

If the primary objective of a study is to test the null hypothesis of no effects, the results of this investigation suggest that there is little to be gained in going to the extra effort of using statistical tests that take the error structure into account since score tests based on the measured doses $x$, without adjustment, do nearly as well. These results should not be interpreted as indicating that results based on $x$ will do as well as those based on the true doses $z$. If the option of measuring doses more precisely is available, this would generally increase power.

(a) Clayton D. C. In press. "Models for the Analysis of Cohort and Case-Control Studies with Inaccurately Measured Exposure." In: Longitudinal methods in Health Research. 0xford: Oxford University Press. 
For this investigation, the probability density functions of the true doses as well as of the measured doses conditional on the true doses have been assumed to be continuous regularly behaved functions. In the real world, measurement errors may result from many sources and may not behave in this manner. In particular, very large measured doses, or "outliers, " may often result from measurement errors that do not follow regular patterns. Results examining the effects of truncation (Tables 9 and 10), and also comparison of results for L1 and G1 with the "longer tailed" LI' and G2' indicate that the the nature of the tails of the distributions as well as the manner in which large doses are handled can have a strong effect on results, especially for the log-linear model. The question of the impact of outliers and how they should be handled in analysis is one that merits further investigation. Recently developed diagnostic techniques (Storer and Crowley 1985) may play an important role here.

This paper has presented asymptotic results. A simulation study evaluating results of the fitting of linear and log-linear models for various error structures with moderate sample sizes would be of interest. In some cases, the distorting effects of measurement error may be unimportant relative to usual sampling variation. For example, it seems unlikely that the distortion in the shape of the dose response curve (see Figures 2 and 3) with moderate errors would be detected with modest sample sizes. In addition, a simulation study could determine the actual distribution of deflation factors that would be expected under repeated sampling. It is possible, for example, that in some instances, much of the distorting effect is due to a few large measured exposures that occur with very small probability. 


\subsection{REFERENCES}

Cochran W. G. 1965. "Errors of Measurement in Statistics." Technometrics $10: 637-665$.

Copeland K. T., H. Checkoway, A. J. McMichael, and R. H. Holbrook. 1977.

"Bias Due to Misclassification in the Estimation of Relative Risk." Am. J. Epidemiol. 105:488-495.

Cox D. R. 1972. "Regression Models and Life Tables." J. R. Statist. Soc. B. $34: 187-220$.

Flegel K. M., C. Brownie, and J. P. Haas. 1986. "The Effects of Exposure Misclassification on Estimates of Relative Risk." Am. J. Epidemiol. $123: 736-51$.

Gilbert, E. S. 1984. "Some Effects of Random Dose Measurement Errors on Analysis of Atomic Bomb Survivor Data." Radiat. Res. 98:591-605.

Gilbert E. S. 1985. "How Much Can Be Learned From Populations Exposed to Low Levels of Radiation?" The Statistician 34:133-44.

Gladen B., and W. J. Rogan. 1979. "Misclassification and the Design of Environmental Studies." Am. J. Epidemiol. 109:607-16.

Jablon S. 1971. Atomic Bomb Radiation Dose Estimation at ABCC. ABCC TR 23-71. AvailabTe from Radiation Effects Research Foundation, 5-2 Hijiyama Park, Hiroshima 730, Japan.

Johnson N. L., and S. Kotz. 1970. Continuous Univariate Distributions - 1. Houghton Miffl in Co. Boston, Massachusetts, pp. 112-119, 166-71.

Kalbfleisch J. D., and R. L. Prentice. 1980. The Statistical Analysis of Failure Time Data. John Wiley and Sons, New York, pp. 44-45.

Kato H., and W. J. Schull. 1982. "Studies of the Mortality of A-bomb Survivors. 7. Mortality 1950-1978: Part I Cancer Mortality." Radiat. Res. 90:395-432.

Kendall M. G. and A. Stuart 1979. The Advanced Theory of Statistics, Vol. 2, 4th Ed., MacMillan, New York, pp. 256-257, 413-415.

Marshal J. R., R. Priore, S. Graham, and J. Brasure. 1981. "On the Distortion of Risk Estimates in Multiple Exposure Level Case-control Studies." Am. J. Epidemiol. 113:464-473.

Prentice R. L. 1982. "Covariate Measurement Errors and Parameter Estimation in Cox's Failure Time Regression Model." Biometrika 69:331-42.

Prentice R. L., and M. W. Mason. 1986. "On the Application of Linear Relative Risk Models." Biometrics 42:09-120. 
SAS Institute Inc. 1982. SAS User's Guide, Basics, 1982 Edition. SAS Institute Inc., Cary, North Carolina.

Storer B. E., and J. Crowley. 1985. "A Diagnostic for Cox Regression and General Conditional Likelihood." J. Am. Statist. Assoc. 80:139-147.

Thomas D. C. 1981. "General Relative Risk Models for Survival Time and Matched Case-control Analysis." Biometrics 37:673-86.

Whittemore A. S., and S. Grosser. 1986. "Regression Methods for Data with Incomplete Covariates." In: Modern Statistical Methods in Chronic Disease Epidemiology, eds. S. H. Moolgakvar, R. L. Prentice, pp. 19-34, John Wiley and Sons New York. 
APPENDIX

TECHNICAL DETAIL AND COMPUTATIONAL PROCEDURES 


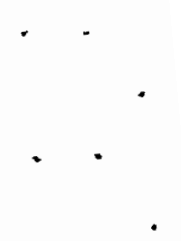




\section{APPENDIX}

\section{TECHNICAL DETAIL AND COMPUTATIONAL PROCEDURES}

\section{A.1 INFERENCES BASED ON LINEAR AND LOG- LINEAR PROPORT IONAL HAZARDS MODELS}

All expressions are initially given in terms of the true doses $z$; specific applications for doses measured with error are given in the sections that follow. Kalbfleish and Prentice (1980) provide additional material on the justification and application of these procedures.

The partial log-likelihoods of interest, with a single covariate, are given by

$$
\sum_{i=1}^{n}\left\{\log \left(1+\beta z_{i}\right)-\log \sum_{u \in R_{i}}\left(1+\beta z_{u}\right)\right\}
$$

for the linear hazard of the form $\lambda_{t}(1+\beta z)$

and by

$$
\sum_{i=1}^{n}\left\{\beta z_{i}-\log \sum_{u \in R_{i}} \exp \left(\beta z_{u}\right)\right\}
$$

for the $\log -1$ inear hazard of the form $\lambda_{t} \exp (B z)$, where the sum $i$ is over the cases of disease that have occurred, and $R_{i}$ denotes the set of doses of those still at risk at the time that the case occurred. Censoring is allowed for since subjects can drop out of the risk sets for reasons other than the disease of interest. In the body of the paper, it was assumed that the distribution of $z$ did not change over time and could be represented as a continuous distribution. Thus these equations can be written as

$$
\sum_{i=1}^{n} \log \left(1+\beta z_{i}\right)-n \log [1+\beta E(z)]
$$


and

$$
\beta \sum_{i=1}^{n} z_{i}-n \log E[\exp (\beta z)]
$$

where $E(z)=\int z g_{z}(z) d z, E[\exp (\beta z)]=\int \exp (\beta z) g_{z}(z) d z, g_{z}(z)$ is the marginal density of $z$.

The likelihood equations to be solved for $\hat{\beta}$ are given by

$$
\begin{aligned}
& \sum_{i=1}^{n} \frac{z_{i}}{1+\hat{\beta} z_{i}}-n \frac{E(z)}{1+\hat{\beta} E(z)}=0 \\
& \sum_{i=1}^{n} z_{i}-n \frac{\int g_{z}(z) z \exp (\hat{\beta} z) d t}{E[\exp (\hat{\beta} z)]}=0,
\end{aligned}
$$

while the efficient score test for testing the null hypothesis that $\beta=0$ is based on the statistic

$$
\sum_{i=1}^{n} z_{i}-n E(z)
$$

for both the linear and log-linear models.

\section{A.2 OPTIMAL PROCEDURE WHEN THE ERROR STRUCTURE CAN BE SPECIFIED}

Prentice (1982) has shown that under assumptions stated earlier, appropriate inferences for a linear model based on the measured $x$ are obtained by replacing $x$ by $E(z ; x)$, while for the log-linear model, they are obtained by replacing $\exp (B x)$ by $E[\exp (B z) ; x]$. In this paper, the error structure is specified in terms of $g_{z}(z)$, the marginal density of $z$, and $f(x ; z)$, the conditional density of $x$ given $z$. Applying Bayes Theorem, we can write 


$$
E(z ; x)=\frac{\int z f(x ; z) g_{z}(z) d z}{\int f(x ; z) g_{z}(z) d z}
$$

and $\quad E[\exp (\beta z) ; x]=\frac{\int \exp (\beta z) f(x ; z) g_{z}(z) d z}{\int f(x ; z) g_{z}(z) d z}$

\section{A.3 ASYMPTOTIC RELATIVE EFFICIENCY}

Kendall and Stuart (1979) have shown that the asymptotic relative efficiency (ARE) of a procedure based on test statistic $Y_{j}$ relative to a test based on $Y_{j}{ }^{\prime}$, (for testing $\left.\beta=0\right)$ is given by $\left(c_{j} / c_{j 1}\right)^{2}$ where

$$
c_{j}=E_{j}^{\prime}(0) /\left[\operatorname{Var}\left(Y_{j}\right) \mid B=0\right]^{1 / 2}
$$

and $E^{\prime}(0)$ is the first derivative of the expected value of $Y_{j}$ with respect to $\beta$ evaluated at $\beta=0$. We are interested in comparing the score test based on measured doses $x$ with that based on true doses $z$, and with that based on $E(z ; x)$, the optimal test if the error structure can be specified. The test statistic based on $z$ is given by (A3), while the other test statistics of interest can be obtained by substituting $x$ or $E(z ; x)$, respectively, for $z$ in equation (A3).

In all cases the expected value of $Y_{j}$ will be given by

$$
E\left(Y_{j}\right)=n \int g_{v}(v) P(v)[v-E(v)] d v
$$

where $v$ may be $z, x$ or $E(z ; x)$, where $g_{v}(v)$ denotes the marginal density for $v$, and $P(v)$ denotes the conditional probability that the value $v$ was selected as the case given that a case occurred. When $v=z, P(v)$ will be given by

$$
\frac{1+\beta z}{1+\beta E(z)} \text { and } \frac{\exp (\beta z)}{E[\exp (\beta z)]}
$$

for the linear and log-linear models, respectively. When $v$ equals $x$ or $E(z ; x), P(v)$ will instead be given by

$$
\frac{1+\beta E(z ; x)}{1+\beta E(z)} \text { and } \frac{E[\exp (\beta z) ; z]}{E[\exp (\beta z)]} \text {, respectively. }
$$


For both linear and log-linear models, the first derivative of $E(Y)$ with respect to $\beta$ and evaluated at $\beta=0$ can be shown to be $\operatorname{Var}(z), \operatorname{Cov}[E(z ; x), x]$, and $\operatorname{Var}[E(z ; x)]$, for $v=z, x$, and $E(z ; x)$, respectively. It can also be shown, using basic properties of conditional expectations, that $\operatorname{Cov}[E(z ; x), x]$ $=\operatorname{Cov}(z, x)$. In addition, it is readily shown that $\operatorname{Var}(Y ; \beta=0)=\operatorname{Var}(v)$. Thus the asymptotic relative efficiency of a test based on the measured doses relative to that based on the true doses is given by $\operatorname{Cov}^{2}(x, z) /[\operatorname{Var}(x) \operatorname{Var}(z)]$.

If $E(x ; z)=b z$, as is the case with the error distributions used in this paper, it is can be shown that $\operatorname{Cov}(x, z)=\operatorname{bVar}(z)$ so that the expression for the asymptotic relative efficiency becomes $b^{2} \operatorname{Var}(z) / \operatorname{Var}(x)$. Since the error parameters ( $h, k$, and $s)$ were chosen so that $\operatorname{Var}(x / b)$ is either $100 \%$ or $20 \%$ larger than $\operatorname{Var}(z)$, it follows that the asymptotic relative efficiencies corresponding to these choices will be approximately $50 \%$ and $83 \%$, respectively. These values are an approximation because of the truncation of negative values noted in the body of the paper. The asymptotic relative efficiency of the test based on $x$ relative to the optimal test based on $E(z ; x)$ will be given by $\operatorname{Cov}^{2}(z, x) /\{\operatorname{Var}[E(z ; x)] \operatorname{Var}(x)\}$.

\section{A.4 DISTORTION OF THE SHAPE OF THE DOSE RESPONSE FUNCTION}

As noted in the text, this evaluation requires the evaluation of $E(z ; x)$ and of $E[\exp (B x) ; z]$ as defined in equations (A4) and (A5). A quantitative measure of the conformity of $E(z ; x)$ to linearity can be obtained by calculating the squared correlation coefficient of $E(z ; x)$ and $x$. Since $\operatorname{Cov}[E(z ; x), x]=\operatorname{Cov}(z, x)$, this squared correlation coefficient is the same as the expression for the asymptotic relative efficiency of the score test based on $x$ relative to that based on the optimal $E(z ; x)$. Since Prentice (1982) has shown that the use of $x$ instead of $E(z ; x)$ will provide an optimal test if and only if $E(z ; x)$ is a linear function of $x$, this equivalence might have been anticipated.

\section{A.5 BIAS IN THE ESTIMATED REGRESSION COEFF ICIENTS}

If measurement error is not accounted for in the fitting of proportional hazards models, the likelihood equations to be solved for linear and log-linear models, respectively, are those given in equations (A1) and (A2), but with the measured $x$ instead of $z$. 
The expected value, taken over $x$ of these likelihood equations can be written as

$$
\int g_{x}(x)[1+\beta E(z ; x)]\left\{\frac{x}{1+\hat{\beta} x}-\frac{E(x)}{1+\beta E(x)}\right\} d x=0
$$

for the linear model, and as

$$
\int g_{x}(x) E[\exp (\beta z) ; x]\left\{x-\frac{\int f_{x}(x) x \exp (\hat{\beta} x) d x}{\int f_{x}(x) \exp (\hat{\beta} x) d x}\right\} d x=0
$$

for the log-linear model. The factor by which the estimated regression coefficients can be expected to be biased downward can be obtained by solving the above equations for $\hat{B}$, and then calculating $D=\beta / \hat{B}$.

\section{A.6 ASYMPTOTIC VARIANCES OF THE ESTIMATED PARAMETERS}

Asymptotically, the variance of the estimated parameters can be obtained as the reciprocal of the expected information. If measurement error is not accounted for, the information would be obtained as the second derivative of the log-likelihood based on $x$, and the expected information per failure would be obtained using the expressions below for the linear and log-linear models, respectively.

$$
\begin{aligned}
& \int g_{x}(x) \frac{1+\beta E(z ; x)}{1+\beta E(z)}\left\{\frac{x^{2}}{(1+\beta x)^{2}}-\frac{E^{2}(x)}{[1+\beta E(x)]^{2}}\right\} d x \\
& \int g_{x}(x) \frac{E[\exp (\beta z) ; x]}{E[\exp (\beta z)]}\left\{\frac{E\left[x^{2} \exp (\beta x)\right]}{E[\exp (\beta x)]}-\frac{E^{2}[x \exp (\beta x)]}{E^{2}[\exp (\beta x)]}\right\} d x
\end{aligned}
$$

If the appropriate procedure given by Prentice (1982) were used, then the information for the linear model would be given by (A8) with $x$ replaced by $E(z ; x)$. For the $10 \mathrm{~g}-1$ inear model, determining the appropriate information requires the first and second derivatives of $E[\exp (\beta z) ; x)]$. If it is assumed 
that differentiation and integration can be interchanged, then the expected information per failure can be shown to be equal to

$$
\begin{gathered}
\frac{\int g_{z}(z) z^{2} \exp (\beta z) d z}{E[\exp (\beta z)]}-\frac{\left[\int g_{z}(z) z \exp (\beta z) d z\right]^{2}}{E^{2}[\exp (\beta z)]} \\
-\int g_{x}(x) \frac{E[\exp (\beta z) ; x]}{E[\exp (\beta z)]}\left\{\frac{E\left[z^{2} \exp (\beta z) ; x\right]}{E[\exp (\beta z) ; x]}-\frac{E^{2}[z \exp (\beta z) ; x]}{E[\exp (\beta z) ; x]}\right\} d x
\end{gathered}
$$

Thus the reciprocals of these expressions would give the correct asymptotic variance of the estimated parameter if the appropriate procedure for handling measurement had been applied. Since, when measurement error is not accounted for, the estimated parameter is biased downward by a factor D, the "correct" asymptotic variance in this case would be given by these reciprocals multiplied by $D^{2}$.

\section{A.7 COMPUTATIONAL PROCEDURES}

The expressions requiring evaluation in this paper are all based on expectations over the joint distribution of $x$ and $z$, which can be specified as $f(x ; z) g_{z}(z)$, where $g_{z}(z)$ denotes the marginal density of $z$, and $f(x ; z)$ denotes the conditional density of $x$ given $z$. As noted in the text, these densities have been taken to be lognormal, gamma or normal densities.

Such expressions can be approximated by replacing the integrals by sums, obtained by choosing cutpoints $c_{1}, c_{2}, \ldots, c_{n}$ such that $\ln \left(c_{k+1}\right)-$ $\ln \left(c_{k}\right)=\delta$, and defining $y_{k}$ as the geometric mean of $c_{k}$ and $c_{k+1}, c_{0}=0$, $y_{0}=0.5 c_{1}$. The discrete approximation to the density for $z$ is then given by

$g_{z}^{\star}(z)=\int_{c_{k}}^{c^{k+1}} g_{z}(z) d z=q_{k}$ for $z=y_{k}, k=0, \ldots, n$ and zero elsewhere. The discrete approximation for the densities of $x$ conditional on $z$ is given by $f *\left(x ; z_{k}\right)=\int_{c_{j}}^{c} j+1\left(x ; z_{k}\right) d x=g_{j k}$ for $x=y, j=0, \ldots, n$ and zero el sewhere. 
For the lognormal true dose densities, $\delta=\sigma / 4$, where $\sigma$ is the standard deviation of $\log z$. The first cutpoint $c_{1}$ is taken to be $\xi-5^{\star} \sigma$, where $\xi$ is the mean of $\log z$. For the gamma densities, the same values were used for $\delta$ and $c_{1}$ as would have been used for a lognormal density with the same coefficient of variation. For the normal error densities I and II,

$$
h *\left(0 ; z_{k}\right)=\int_{-\infty}^{0} g\left(x ; z_{k}\right)=P_{0 k} .
$$

These integrals were evaluated using the SAS normal and gamma probability functions PROBNORM and PROBGAM, respectively (SAS Institute Inc. 1982).

The expressions $E(z ; x)$ and $E[\exp (B z) ; x]$ can be written as indicated in equations (A4) and (A5). The numerators can be approximated by

$$
\begin{aligned}
& \sum_{k=0}^{n} y_{k} p_{j k} q_{k} \quad \text { or } \sum_{k=0}^{n} \exp \left(\beta y_{k}\right) p_{j k} q_{k} \\
& \text { and the denominator by } \sum_{k=1}^{n} p_{j k} q_{k}=p_{j} \text {. }
\end{aligned}
$$

The value of $n$ is chosen so that the contribution to these sums for terms with $k, j>n$ is negligible $(<0.000001)$.

$\operatorname{Var}[E(z ; x)]$, which is required for the asymptotic relative efficiency, can be approximated by

$$
\sum_{j} p_{j} E^{2}\left(z ; x_{j}\right)-\left[\sum_{j} p_{j} E\left(z ; x_{j}\right)\right]^{2}
$$

where $p_{j}$ and $E\left(z ; x_{j}\right)$ have been evaluated as indicated in (A11) and $(A 12)$. $\operatorname{Var}(x)$ can be approximated in a similar manner substituting $x_{j}$ for $E\left(z ; x_{j}\right)$ in the above expression.

The factors $D$ are obtained by replacing the integrals in equations (A6) and (A7) with sums and then using a Newton Raphson procedure to solve for $\hat{\beta}$. The "estimated" and "correct" variances can be calculated by substituting sums for integrals in expressions (A8), (A9), and (A10). 

PNL -6578

UC-605

DISTRIBUTION

No. of

Copies

OFFSITE

Robert Goldsmith, Ph.D.

U.S. Department of Energy

Office of Health and

Environmental Research

Washington, DC 20545

10 Office of Scientific and

Technical Information

ONSITE

DOE Richland Operations Office

B. C. Norman

39 Pacific Northwest Laboratory

W. J. Bair

E. S. Gilbert (30)

J. A. Mahaffey

Publishing Coordination (2)

Technical Report Files (5) 


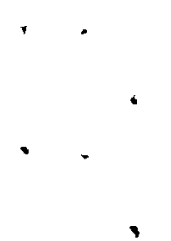

